\title{
Recommendations by Canadian urologists and radiation oncologists for the treatment of clinically localized prostate cancer
}

\author{
Andrew Pearce, MSc, MD; Chris Newcomb, PhD; Siraj Husain, MD
}

See related article on page 204

\section{Abstract}

Objective: Previous work has shown that urologists and radiation oncologists prefer the treatment that they themselves deliver when treating clinically localized prostate cancer. Our objective was to determine whether Canadian radiation oncologists and urologists have similar biases in favour of the treatments that they themselves deliver for localized prostate cancer.

Methods: We developed a survey to poll the beliefs that Canadian radiation oncologists and urologists held toward prostate specific antigen (PSA) screening, survival benefits of treatment, recommendations for treatment of prostate cancer and the likelihood of side effects with each therapy.

Results: Urologists were more likely to recommend routine PSA screening for men up to age $70(p<0.001)$, while radiation oncologists were more likely to recommend PSA screening for men over age $80(p<0.04)$. More urologists felt that there was "definitely" a survival advantage with radical prostatectomy (RP) $(60 \%$ v. $21 \%, p<0.001)$. More radiation oncologists recommend external beam radiation therapy (EBRT) $(p<0.01)$ or brachytherapy $(p<0.03)$ to treat low-risk prostate cancer. More urologists than radiation oncologists recommend RP for intermediate-risk patients $(98 \%$ v. $70 \%, p<0.001)$.

Conclusion: Most Canadian urologists and radiation oncologists recommend routine PSA screening for men aged 50 to 70. A significant preference was detected among both urologists and radiation oncologists for the treatment that they themselves deliver. While both urologists and radiation oncologists recommend prostatectomy for the treatment of low-risk localized prostate cancer, urologists are significantly less likely to recommend EBRT. Conversely, when patients present with intermediate-risk prostate cancer, radiation oncologists were significantly less likely than urologists to recommend a prostatectomy.

CUAJ 2008;2(3):197-203

\section{Introduction}

Several treatment modalities are currently available to treat early-stage prostate cancer. Depending on patient preferences and comorbidities, a selection may be made between watchful waiting, radical prostatectomy $(\mathrm{RP})$, external beam radiation therapy (EBRT), brachytherapy and cryotherapy. It is likely that the treatment recommendation given to patients by their specialist will play an important role in their decision-making process. Previous work has shown that urologists and radiation oncologists prefer the treatment that they themselves deliver. A 1988 survey by Moore and colleagues ${ }^{1}$ demonstrated that should they themselves be diagnosed with localized prostate cancer, $79 \%$ of urologists would elect to undergo RP while $92 \%$ of radiation oncologists would choose EBRT. A survey published in JAMA in 2000 demonstrated that although 93\% of American urologists felt that RP was the preferred treatment option for localized prostate cancer, $72 \%$ of radiation oncologists felt that RP and EBRT were equivalent treatments. ${ }^{2}$

Our objective was to determine whether Canadian radiation oncologists and urologists have similar biases in favour of the treatments that they themselves deliver for localized prostate cancer. We also wanted to determine what their attitudes were regarding prostate specific antigen (PSA) screening in comparison with the Canadian Task Force on Preventative Medicine's recommendation against PSA screening. ${ }^{3}$ In addition, we examined the opinions of the 2 groups of specialists regarding the likelihood of survival benefits with and side effects of the various treatment options available for localized prostate cancer.

\section{Methods}

We developed a survey to poll the beliefs that Canadian radiation oncologists and urologists held toward PSA screening, survival benefits of treatment, recommendations for treatment of prostate cancer and the likelihood of side effects with each therapy (Appendix 1).

Each regular member of the Canadian Association of Radiation Oncologists was sent an email with a link to an online survey instrument that collected the data (Survey Monkey, www.surveymonkey.com). A total of 209 functional email addresses were obtained, 86 of which were returned for a $41 \%$ response rate. Six radiation oncologists declined to complete the survey as they did not treat prostate 
cancer. A further 6 radiation oncologists declined to complete the survey without giving a reason. Nonrespondents were not routinely recontacted.

As we were not able to obtain email addresses for Canadian urologists, fax numbers for Canadian urologists were obtained from the College of Physician and Surgeons website from each province. Alberta, Ontario, Nova Scotia and New Brunswick all provided fax numbers for each urologist in their respective provinces. Newfoundland, Quebec, Manitoba, Saskatchewan and British Columbia did not provide fax numbers; however, some urologists were registered with colleges in the other provinces and were contacted. A total of 275 urologists were sent our survey by fax. We received 102 responses from Canadian urologists for a $37 \%$ response rate. Four respondents did not fill out the survey because they practised only pediatric urology and therefore did not treat prostate cancer. Nonrespondents were not routinely recontacted.

All questionnaires faxed back from Canadian urologists were manually entered into the same online survey instrument that was used to collect the radiation oncologist data. The online survey instrument was used to tabulate the data. All statistical analysis was performed using the statistical package contained within Microsoft Excel (Microsoft Corp., Redmond, Wash.). All $p$ values quoted are 2-sided Student $t$ tests.

\section{Results}

\section{Demographics}

The majority of polled radiation oncologists were

\begin{tabular}{|c|c|c|c|}
\hline \multirow[b]{2}{*}{ Variable } & \multicolumn{2}{|c|}{ Group, no. (and \%) } & \multirow[b]{2}{*}{$p$ value } \\
\hline & $\begin{array}{l}\text { Radiation } \\
\text { oncologists }\end{array}$ & Urologists & \\
\hline \multicolumn{4}{|l|}{ Type of remuneration } \\
\hline Salary & $35(49)$ & $5(5)$ & $<0.001$ \\
\hline Fee for service & $3(4)$ & $84(83)$ & $<0.001$ \\
\hline Mixed & $34(47)$ & $12(12)$ & $<0.001$ \\
\hline \multicolumn{4}{|l|}{ Treatment offered } \\
\hline Treats prostate cancer & $48(66)$ & $91(90)$ & $<0.001$ \\
\hline $\begin{array}{l}\text { Performs brachytherapy } \\
\text { implants }\end{array}$ & $22(31)$ & $6(6)$ & $<0.001$ \\
\hline
\end{tabular}

remunerated on a salary basis $(49 \%)$ or a mixed salary and fee-for-service model (47\%). In contrast, the majority of urologists were remunerated on a fee-for-service basis (83\%) (Table 1). More urologists than radiation oncologists treat prostate cancer as a regular part of their practice $(90 \% \mathrm{v}$. $66 \%$ ). Brachytherapy implants were performed by more radiation oncologists than urologists $(31 \%$ v. $6 \%$ ) (Table 1).

\section{PSA screening}

A significantly larger percentage of urologists recommend routine PSA screening for men aged 50 years and under up to age $70(p<0.001)$ (Fig. 1). An equal number of specialists recommend PSA screening for men aged 70-80 years. More radiation oncologists than urologists recommend PSA screening for men over age $80(20 \%$ v. $6 \%, p<0.04)$.

\section{Survival benefits}

We detected significant differences between the groups when they were asked "do you feel that there is a survival benefit to treating low risk prostate cancer (cT2A or below, Gleason 6 or less, PSA $10.0 \mathrm{ng} / \mathrm{mL}$ or less) in patients with a life expectancy of more than 10 years?" With regard to RP, more urologists felt that there was "definitely" a survival advantage (60\% v. $21 \%, p<0.001)$ (Fig. 2). Conversely, more radiation oncologists felt that there was no survival advantage to RP in this group $(12 \% \mathrm{v} .1 \%, p<0.02)$ (Fig. 2$)$. When the conditions were changed so that the patient's life expectancy was less than 10 years, there were

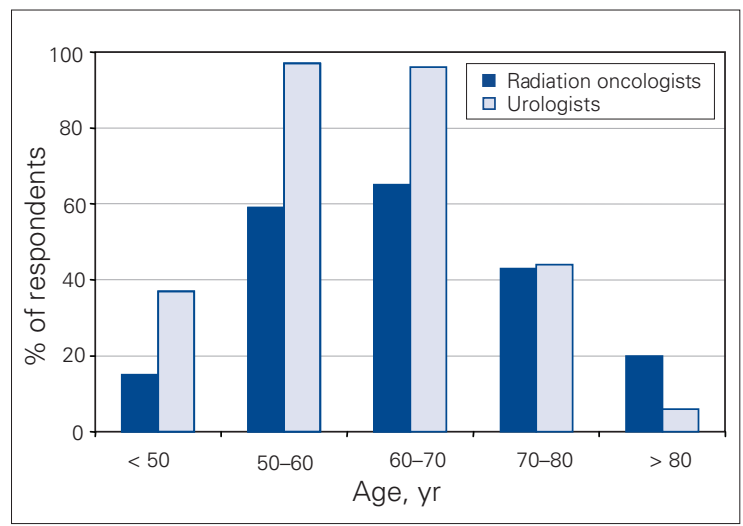

Fig 1. Percentage of specialist respondents who would recommend prostate specific antigen screening. 
no significant differences between the opinions of radiation oncologists and urologists, with $78 \%$ of radiation oncologists and $80 \%$ of urologists believing that there was no survival advantage. When asked the above question regarding patients with a greater than 10 year life expectancy, using cryotherapy as the treatment modality, radiation oncologists were more likely to feel that there was not a survival benefit $(75 \%$ v. $50 \%, p<0.03)$ (Fig. 2). Neither group felt that there was a survival advantage to cryotherapy for patients who had a life expectancy of less than 10 years $(94 \%$ radiation oncologists, $92 \%$ urologists). Both groups felt that there was "probably" a survival advantage to treating with brachytherapy for patients with a life expectancy of more than 10 years $(75 \%$ radiation oncologists, $71 \%$ urologists) and that there was no survival advantage for patients with a life expectancy of less than 10 years $(76 \%$ radiation oncologists, $80 \%$ urologists). Both groups also felt that there was "probably" a survival advantage to treating with EBRT for patients with a life expectancy of more than 10 years $(73 \%$ radiation oncologists, $62 \%$ urologists), but that there was no survival advantage for patients with a life expectancy of less than 10 years $(78 \%$ radiation oncologists, $76 \%$ urologists).

\section{Low-risk treatment recommendations}

Participants were then asked, "which treatment would you recommend to a patient with prostate cancer, cT2A, Gleason 6, PSA 6 in a patient with a life expectancy of more than 10 years and no

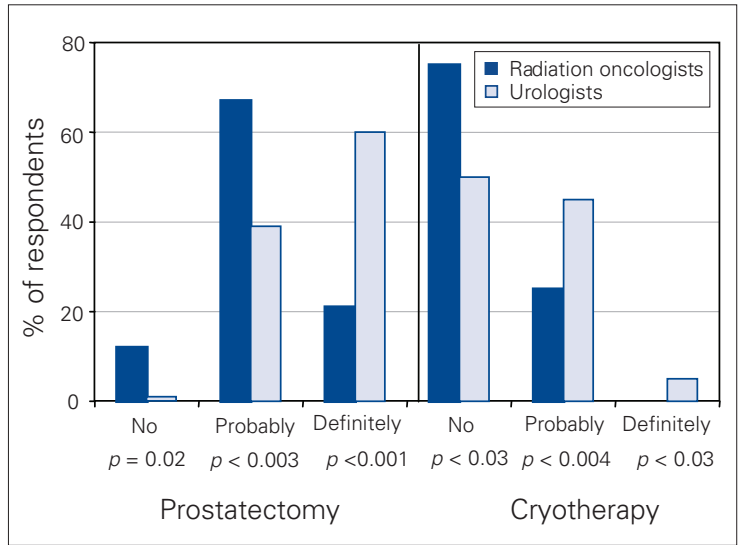

Fig 2. Respondents' opinions regarding survival advantage of treating low-risk prostate cancer in patients with a $>10$ year life expectancy. contraindications to any therapy?" The list of recommended treatment options from which they could choose included watchful waiting, androgen suppression alone, prostatectomy, cryotherapy, brachytherapy and EBRT. Respondents were asked to indicate all options that they would recommend equally. Forty-nine percent of radiation oncologists and $44 \%$ of urologists were comfortable recommending watchful waiting for such a patient. Nether group recommended androgen suppression alone $(1.5 \%$ radiation oncologists, $0 \%$ urologists). Only $6 \%$ of radiation oncologists and $8 \%$ of urologists recommended cryotherapy. Brachytherapy was a more popular treatment option among radiation oncologists than urologists ( $89 \%$ v. $67 \%, p<0.03)$. Both groups felt that $\mathrm{RP}$ was a reasonable treatment option (88\% radiation oncologists, 92\% urologists). EBRT was recommended by more radiation oncologists than urologists $(82 \%$ v. $56 \%, p<0.01)$ (Fig. 3 ).

\section{Intermediate-risk treatment recommendations}

To assess how the 2 specialist groups felt about treating patients who would be considered at intermediate risk for extraprostatic extension by the Canadian consensus guidelines, ${ }^{4}$ we asked the following question: "Which treatment would you recommend to a patient with prostate cancer, CT2B, Gleason 7, PSA 11, in a patient with a life expectancy of more than 10 years and no contraindications to any therapy?" The same 6 treatment options were presented to respondents as in the previous question. Only a minority of respondents in each group felt that watchful waiting,

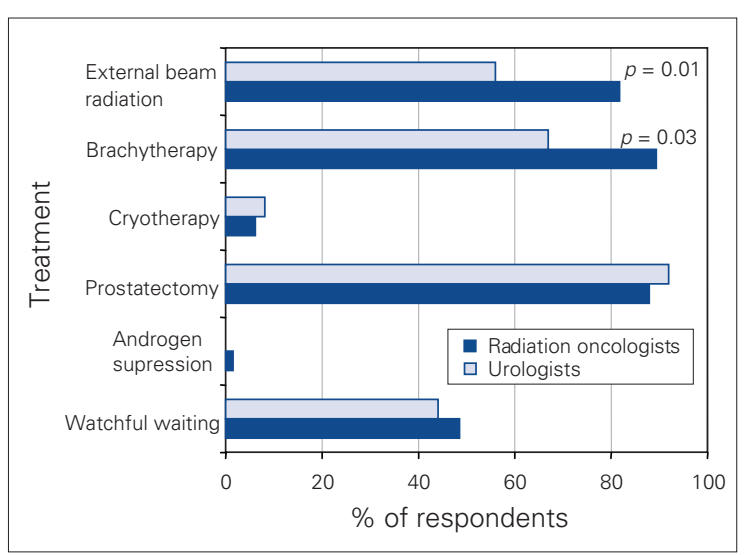

Fig 3. Respondents' recommendations for treating patients with low-risk prostate cancer. 
androgen suppression alone or cryotherapy were appropriate treatment options (Fig. 4). Significantly more urologists than radiation oncologists felt that RP was an appropriate treatment for such a patient $(98 \%$ v. $70 \%, p<0.001)$ (Fig. 4). More radiation oncologists than urologists felt that brachytherapy (25\% v. $10 \%, p<0.04)$ and EBRT (94\% v. $74 \%)$ was an appropriate treatment option; however, the EBRT difference did not meet statistical significance.

\section{Erectile dysfunction}

When questioned about the likelihood of different treatments causing erectile dysfunction, most urologists and radiation oncologists felt that non-nerve-sparing RP would cause erectile dysfunction "such that erections sufficient for penetration are no longer possible without medication within 3 years" in $75 \%-100 \%$ of patients (70\% urologists, $63 \%$ radiation oncologists). Regarding nerve-sparing RP, the majority of both groups felt that $40 \%-75 \%$ of patients would suffer from erectile dysfunction $(61 \%$ radiation oncologists, $67 \%$ urologists). Fifty percent of each group felt that cryotherapy would cause erectile dysfunction in more than $50 \%$ of patients. Regarding brachytherapy, the majority of both groups felt that $30 \%-50 \%$ of patients were likely to experience this complication (65\% radiation oncologists, $52 \%$ urologists). More urologists than radiation oncologists felt that greater thjan $50 \%$ of patients would have erectile dysfunction with EBRT $(36 \%$ v. $18 \%, p<0.007)$.

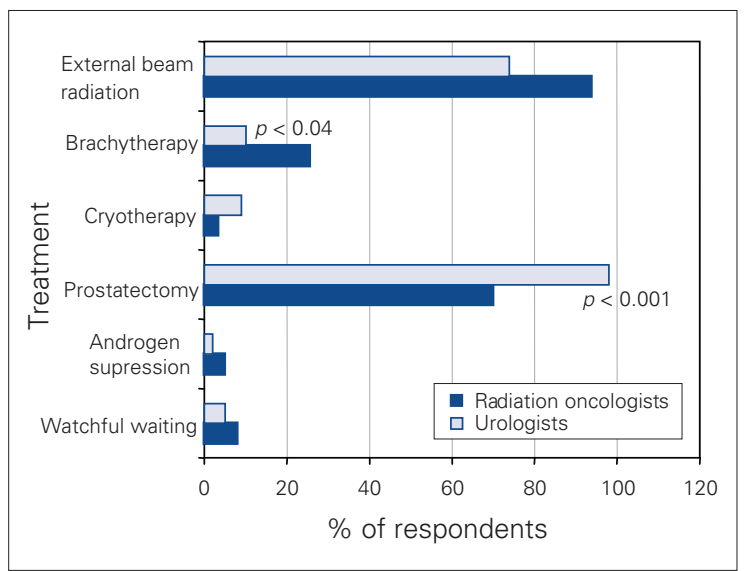

Fig 4. Respondents' recommendations for treating patients with intermediate-risk prostate cancer.

\section{Urinary dysfunction}

Urologists were more likely than radiation oncologists to feel that RP would produce "urinary dysfunction defined as requiring a pad indefinitely" in only $0 \%-10 \%$ of patients (urologists $56 \%$, radiation oncologist 29\%, $p<0.001$ ) (Fig. 5). Most radiation oncologists $(74 \%)$ felt that urinary dysfunction would occur in up to $20 \%$ of patients. The majority of radiation oncologists and urologists felt that cryotherapy would produce urinary incontinence in up to $20 \%$ of patients (radiation oncologists $74 \%$, urologists $78 \%$ ). Most urologists and radiation oncologists felt that brachytherapy and EBRT would produce urinary dysfunction in only $0 \%-10 \%$ of patients.

\section{Bowel dysfunction}

Neither specialist group felt that any therapy would cause "bowel dysfunction defined as rectal bleeding requiring intervention or rectal incontinence requiring a pad indefinitely" in more than $20 \%$ of patients.

\section{Discussion}

Although urologists and radiation oncologists are both extensively involved in the treatment of prostate cancer, previous work has shown that there are differences of opinion regarding appropriate treatment of clinically localized prostate cancer between these 2 groups. Currently, the Canadian Task Force on Preventive Health Care

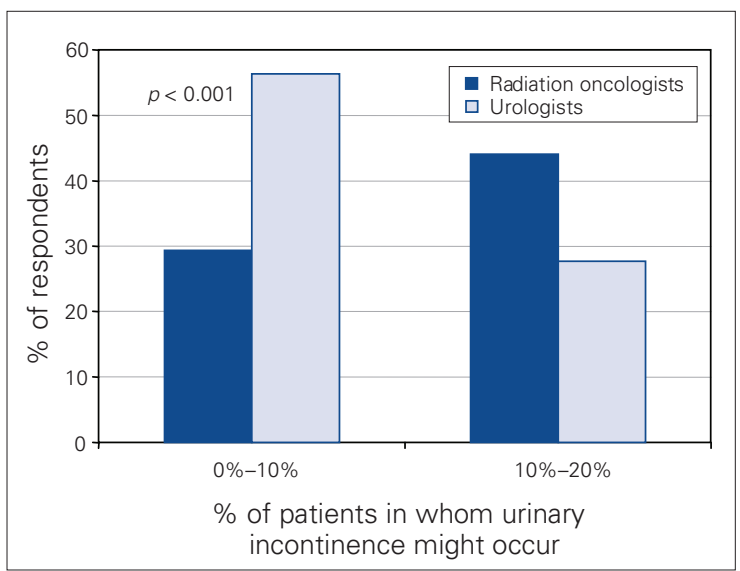

Fig 5. Percentage of patients in whom respondents believed urinary incontinence might occur following radical prostatectomy. 
recommends against PSA screening on the basis of a low positive predictive value and "known risk of adverse affects associated with therapies of unproven effectiveness." ${ }^{\prime 3}$ This recommendation is clearly rejected by both Canadian urologists and radiation oncologists, who both recommend PSA screening especially for men 50 to 70 years old (Fig. 1). Overall, Canadian urologists were more likely to recommend PSA screening for men aged les than 50 to 70 years, while radiation oncologists were more likely to recommend PSA screening for men over age 80. Previous work by Fowler and colleagues ${ }^{2}$ in the United States also showed that American radiation oncologists were more likely than urologists to recommend PSA screening for men over the age of 80 (43\% v. 16\%). There may in fact be a lack of benefit for screening in men over age 75. According to the US Social Security Administration, the life expectancy for all men at age 75 is just under 10 years $\left(9.99\right.$ yr). ${ }^{5}$ Our own study shows that about $80 \%$ of Canadian specialists do not believe that there is a survival advantage to treatment of low-risk prostate cancer if the patient's life expectancy is less than 10 years. At least 1 large randomized screening trial attempting to assess the efficacy of screening has also established an upper age cut-off of 74 years. ${ }^{6}$ Interestingly, the lower percentage of radiation oncologists recommending PSA screening for younger men was not observed among American specialists. For example, $90 \%$ of American radiation oncologists in that report recommended PSA screening for men aged 50-59 years, compared with $61 \%$ in our study. Similarly, in the Gohagan and colleagues ${ }^{6}$ study $98 \%$ of American radiation oncologists recommended PSA screening for men aged 60-69, compared with $64 \%$ in our study. While the reason for these differences could be multifactorial, 1 major difference that we did detect was a significant difference in the percentage of specialists paid on a fee-for-service basis. In Fowler and colleagues' report, ${ }^{2} 58 \%$ of radiation oncologists were remunerated only on a feefor-service basis, compared with $5 \%$ in our study.

Moore and co-authors ${ }^{1}$ published a report in 1988 that showed that $72 \%$ of urologists would elect to undergo an RP while $92 \%$ of radiation oncologists would choose EBRT if they were diagnosed with clinically localized prostate cancer. Fowler and colleagues ${ }^{2}$ found that $93 \%$ of urologists preferred RP for clinically localized prostate cancer in patients with a life expectancy of greater than 10 years, while $72 \%$ of radiation oncologists felt that RP or EBRT were equivalent treatments. Our results are similar in that both urologists and radiation oncologists were comfortable recommending RP for patients with clinically localized prostate cancer; however, significantly fewer urologists were comfortable recommending EBRT for these patients $(56 \%$ urologists, $82 \%$ radiation oncologists; Fig. 3). This difference may arise from different beliefs regarding the likelihood of a survival advantage for these patients with these 2 treatments. While most radiation oncologists felt that there was "probably" a survival advantage to either treatment (69\% RP, 74\% EBRT), urologists were more comfortable with RP $-60 \%$ thought there was "definitely" a survival advantage to RP and $62 \%$ felt that there was "probably" a survival benefit to EBRT. It is interesting to note that the patient in this theoretical situation would have satisfied the eligibility requirements for the Scandinavian RP versus watchful waiting trial, which demonstrated a $5.3 \%$ disease-specific mortality benefit at 10 years in favour of RP and had randomized patients with T1 or T2 tumours, PSA less than $20 \mathrm{ng} / \mathrm{mL}$ and a life expectancy of greater than 10 years.

The second patient situation presented in our study was one of an intermediate-risk prostate cancer, cT2B, Gleason score of 7 and a PSA level of $11 \mathrm{ng} / \mathrm{mL}$ in a patient with a life expectancy of more than 10 years and no contraindications to any therapy. Such a patient would have a $9 \%-14 \%$ likelihood of his disease being confined to the prostate, according to the updated Partin nomograms. ${ }^{8}$ In this setting, 98\% of urologists recommended RP and only $74 \%$ felt that EBRT was an equally effective treatment (Fig. 4). Conversely, $94 \%$ of radiation oncologists felt that EBRT was an appropriate treatment, with significantly fewer radiation oncologists comfortable with RP (67\%). Fowler and colleagues' work $^{2}$ with American specialists shows an even more striking difference: only $27 \%$ of urologists recommended EBRT for a patient with a Gleason score of 7, a PSA level of $10-20 \mathrm{ng} / \mathrm{mL}$ and prostate cancer; only $1 \%$ of radiation oncologists recommended RP.

In the interest of limiting the length of the questionnaire, a high-risk patient situation was not included in our study of localized prostate cancer. We felt that further lengthening the questionnaire 
would have a detrimental effect on response rates and therefore affect the validity of the results. However, exploring differences in recommendations among Canadian urologists and radiation oncologists for the treatment of high-risk prostate cancer is an interesting area of future research.

Although few significant differences were detected in the opinions of the different specialists regarding the likelihood of treatment-related side effects, urologists were more likely than radiation oncologists to feel that cryotherapy and EBRT would cause erectile dysfunction in a higher percentage of patients. Radiation oncologists, compared with urologists, felt that RP would cause urinary incontinence in more patients (Fig. 5).

A limitations of our study is that in the interest of limiting the length of the questionnaire, we only asked about a limited number of clinical scenarios ( 1 low-risk and 1 intermediate-risk case for treatment recommendations). Also, we were not able to obtain fax numbers for urologists in several Canadian provinces, which somewhat restricted our geographic sampling. However, jurisdictions in western, central and eastern Canada were sampled.

\section{Conclusion}

Most Canadian urologists and radiation oncologists recommend routine PSA screening for men aged $50-70$ years, despite the recommendation by the Canadian Task Force on Preventive Health Care that routine PSA screening not be performed. More urologists than radiation oncologists recommend PSA screening for men under age 70, while more radiation oncologists than urologists recommend PSA screening for men over age 80. A significant preference was detected among both urologists and radiation oncologists for the treatment that they themselves deliver. Although both urologists and radiation oncologists recommend prostatectomy for the treatment of low-risk localized prostate cancer, urologists were significantly less likely to recommend EBRT. Conversely, when patients present with intermediate-risk prostate cancer, radiation oncologists were significantly less likely, compared with urologists, to recommend a prostatectomy. Erectile dysfunction was anticipated to be the most common side effect of any therapy.

From the Department of Radiation Oncology, Tom Baker Cancer Centre, University of Calgary, Calgary, Atla.

This article has been peer reviewed.

Competing interests: None declared.

\section{References}

1. Moore MJ, O'Sullivan BO, Tannock IF. How expert physicians would wish to be treated if they had genitourinary cancer. J Clin Oncol 1988;6:1736-45.

2. Fowler FJ, Collins MM, Albertsen PC, et al. Comparison of recommendations by urologists and radiation oncologists for treatment of clinically localized prostate cancer. JAMA 2000;283:3217-22

3. Canadian Task Force on Preventive Health Care. Available: www.ctfphc.org (accessed 2007 July 20).

4. Lukka H, Warde P, Pickles T, et al. Controversies in prostate cancer radiotherapy: consensus development. Can J Urol 2001;8:1314-22.

5. US Social Security Administration. Acutarial publications: period of life table. 2007. Available: www.socialsecurity.gov/OACT/STATS/table4c6.html (accessed 2007 July 20).

6. Gohagan JK, Prorok P, Hayes R, et al. The Prostate, Lung, Colorectal and Ovarian (PLCO) Cancer Screening Trial of the National Cancer Institute: history, organization, and status. Control Clin Trials 2000; (6 Suppl):251S-72S.

7. Bill-Axelson A, Holmberg L, Ruutu M, et al. Radical prostatectomy versus watchful waiting in early prostate cancer. N Engl J Med 2005;352:1977-84.

8. Partin AW, Mangold LA, Lamm DM, et al. Contemporary update of prostate cancer staging nomograms (Partin tables) for the new millennium. Urology 2001;58:843-8.

Correspondence: Dr. Andrew Pearce, Radiation Oncology Resident, Tom Baker Cancer Centre, University of Calgary, 1331 29th St. NW, Calgary AB T2N 4N2; anpearce@cancerboard.ab.ca 


\section{Appendix 1. Low-risk prostate cancer treatment survey}

1. My specialty is (please circle one):

2. I am remunerated by: salary

3. I treat prostate cancer as a significant percentage of my clinical work:

4. I do brachytherapy implants as part of my clinical practice:

5. I would recommend routine PSA screening in the following aged men:

$\begin{array}{lll}<50 & \text { yes } & \text { no } \\ 50-60 & \text { yes } & \text { no } \\ 60-70 & \text { yes } & \text { no } \\ 70-80 & \text { yes } & \text { no } \\ >80 & \text { yes } & \text { no }\end{array}$

6. Do you feel that there is a survival benefit to treating low-risk prostate cancer (cT2A or below, Gleason 6 or less, PSA $10.0 \mathrm{ng} / \mathrm{mL}$ or less) in patients with a life expectancy of less than 10 years with the following therapies (circle one for each)?

$\begin{array}{llll}\text { Prostatectomy } & \text { no } & \text { probably } & \text { definitely } \\ \text { Cryotherapy (cryosurgery) } & \text { no } & \text { probably } & \text { definitely } \\ \text { Brachytherapy } & \text { no } & \text { probably } & \text { definitely } \\ \text { External beam radiotherapy } & \text { no } & \text { probably } & \text { definitely }\end{array}$

7. Which treatment would you recommend to a patient with prostate cancer, cT2A, Gleason 6, PSA $6 \mathrm{ng} / \mathrm{mL}$ in a patient with a life expectancy of more than 10 years and no contraindications to any therapy (please circle all that you would recommend equally)?
Watchful waiting
Androgen suppression alone
Prostatectomy
Cryotherapy (cryosurgery)
Brachytherapy
External beam radiotherapy

8. Which treatment would you recommend to a patient with prostate cancer cT2B, Gleason 7, PSA $11 \mathrm{ng} / \mathrm{mL}$ in a patient with a life expectancy of more than 10 years and no contraindications to any therapy (please circle all that you would recommend equally)?
Watchful waiting
Androgen suppression alone
Prostatectomy
Cryotherapy (cryosurgery)
Brachytherapy
External beam radiotherapy

9. For a patient with normal sexual function, how likely (\% of patients) is each therapy to cause sexual dysfunction so that erections sufficient for penetration are no longer possible without medication

Prostatectomy (nerve sparing) $\quad 0 \%-10 \% \quad 10 \%-20 \% \quad 20 \%-30 \% \quad 30 \%-40 \% \quad 40 \%-50 \% \quad 50 \%-75 \% \quad 75 \%-100 \%$ Prostatectomy (non-nerve sparing) 0\%-10\% $\quad 10 \%-20 \% \quad 20 \%-30 \% \quad 30 \%-40 \% \quad 40 \%-50 \% \quad 50 \%-75 \% \quad 75 \%-100 \%$ Cryotherapy (cryosurgery) $\quad$ 0\%-10\% $\quad 10 \%-20 \% \quad 20 \%-30 \% \quad 30 \%-40 \% \quad 40 \%-50 \% \quad 50 \%-75 \% \quad 75 \%-100 \%$ Brachytherapy $\quad$ 0\%-10\% 10\%-20\% 20\%-30\% 30\%-40\% 40\%-50\% 50\%-75\% 75\%-100\% External beam radiotherapy $\quad$ 0\%-10\% 10\%-20\% 20\%-30\% 30\%-40\% 40\%-50\% $50 \%-75 \% \quad 75 \%-100 \%$

10. For a patient with normal urinary function, how likely (\% of patients) is each therapy to cause urinary dysfunction defined as requiring a pad indefinitely.

Prostatectomy (nerve sparing) $\quad$ 0\%-10\% $\quad 10 \%-20 \% \quad 20 \%-30 \% \quad 30 \%-40 \% \quad 40 \%-50 \% \quad 50 \%-75 \% \quad 75 \%-100 \%$ Prostatectomy (non-nerve sparing) 0\%-10\% $\quad 10 \%-20 \% \quad 20 \%-30 \% \quad 30 \%-40 \% \quad 40 \%-50 \% \quad 50 \%-75 \% \quad 75 \%-100 \%$ Cryotherapy (cryosurgery) $\quad$ 0\%-10\% $\quad 10 \%-20 \% \quad 20 \%-30 \% \quad 30 \%-40 \% \quad 40 \%-50 \% \quad 50 \%-75 \% \quad 75 \%-100 \%$ Brachytherapy 0\%-10\% $10 \%-20 \% \quad 20 \%-30 \% \quad 30 \%-40 \% \quad 40 \%-50 \% \quad 50 \%-75 \% \quad 75 \%-100 \%$ External beam radiotherapy $\quad$ 0\%-10\% $\quad 10 \%-20 \% \quad 20 \%-30 \% \quad 30 \%-40 \% \quad 40 \%-50 \% \quad 50 \%-75 \% \quad 75 \%-100 \%$

11. For a patient with normal bowel function, how likely ( $\%$ of patients) is each therapy to cause bowel dysfunction defined as rectal bleeding requiring intervention or rectal incontinence requiring a pad indefinitely?

Prostatectomy (nerve sparing) $\quad$ 0\%-10\% 10\%-20\% 20\%-30\% 30\%-40\% 40\%-50\% 50\%-75\% 75\%-100\% Prostatectomy (non-nerve sparing) 0\%-10\% $\quad 10 \%-20 \% \quad 20 \%-30 \% \quad 30 \%-40 \% \quad 40 \%-50 \% \quad 50 \%-75 \% \quad 75 \%-100 \%$ Cryotherapy (cryosurgery) Brachytherapy 0\%-10\% 10\%-20\% 20\%-30\% 30\%-40\% 40\%-50\% 50\%-75\% 75\%-100\% External beam radiotherapy 